\title{
Excitonic Emission of a Triply Charged Quantum Dot Molecule in a Magnetic Field
}

\author{
M. Adames and A. S. Camacho \\ Departamento de Física, Universidad de Los Andes. Cra. IE No. 18A-10. Edificio H., Bogotá, Colombia
}

Received on 8 December, 2005

\begin{abstract}
We calculate the exciton states of a Quantum Dot (QD) and a Quantum Dot Molecule (QDM) in an exact way for single, double and triply charged excitons without and with an applied external magnetic field. For the case of single quantum dot, we reproduce the experimental results reported in [2]. We also calculate the emission energies for the neutral molecule QDM as function of the barrier width between the two dots and we find that the Coulomb interaction splits the emission spectra in case of QDM. Concerning hybridization the triply charged QDM behaves in the same way as the QD, but showing several anticrossings with the lower Landau level of the continuum.
\end{abstract}

Keywords: Charge excitons; Excitons in magnetic fields; Quantum dots

\section{INTRODUCTION}

The concept of dimensionality in semiconductor structures has been extremely successful during the last two decades: technological efforts have focused first on realization of quasitwo-dimensional quantum wells and have then shifted toward the fabrication of quasi-one and quasi-zero-dimensional structures such as quantum wires and quantum dots (QD). An artificial quantum dot molecule (QDM) is formed by two coupled QDs structures located close to each other [1]. The fabrication of such structures on the nanometer scale presents a considerable technological challenge. Self-assembled quantum dots have emerged as one of simplest means of exploiting the physics and device applications. Self-assembly quantum dots are interesting systems in which is possible to study the electronic and excitonic confinement physics and also the interaction between the confined units or quantum dots, gaining information about the self-organization processes. Khaled Karrai et al. [2] showed that QDs in a self-assembled system can also possess electronic states that go far beyond the artificial atom model. These states are a coherent hybridization of localized QD states and extended continuum states: they have no analogue in atomic physics. The states are generated by emission by a photon from a quantum dot. Coherent coupling in quantum dots is interesting for applications as quantum gates, where coherence is important so that quantum information is not lost during the gate operation. Single charge excitations (excitons) in semiconductor quantum dots represent an attractive quantum bit since they can coherently manipulated using ultrafast laser pulses over time scales much shorter than their coherence times. However, our interest is in the coupling between the dots is focused in the hybridization and how these new states could affect the formation of a QDM. We calculate the exciton states of a QD and a QDM quantitatively, in order to obtain the exciton energies in these systems for single, double and triply charge excitons without and in the presence of an external magnetic field. For the QD we reproduce the experimental results reported in [2]. We also calculate the emission energies for the neutral molecule and for the charged QDM as function of the barrier width between the two dots and we find that the Coulomb interaction splits the emission spectra as expected. Additionally, in the triply charged QDM we introduce the hybridization with a Landau level of the continuum and we obtain the coupling with the continuum for the same electronic configuration of the single QD at 6,5 T.

\section{THEORY}

The Hamiltonian of the system is given by

$$
\mathrm{H}=\mathrm{H}_{s p}+\mathrm{H}_{e-e}+\mathrm{H}_{e-h}+\mathrm{H}_{t u n}
$$

where $\mathrm{H}_{s p}$ is the single particle Hamiltonian in the QD [3], $\mathrm{H}_{e-e}$ and $\mathrm{H}_{e-h}$ are the electron-electron and electron-hole interaction energies, respectively [4], and the last term is the tunneling which permits interaction with the Wetting Layer (WL) [5].

For our calculations we suppose a parabolic confinement. We consider here the first two harmonic oscillators states, which are labeled as s and p. At zero magnetic field $B$, the $\mathrm{s}$ level is twofold degenerate and the $\mathrm{p}$ level is fourfold. For $\mathrm{p}$ level the states are classified with the quantum number $\mathrm{m}$, corresponding to eigenvalues of the $\mathrm{L}_{z}$ angular momentum operator, as this is the natural basic to use in a magnetic field applied along the $\mathrm{z}$ direction. In a magnetic field, is convenient to define an effective frecuency $\Omega$ where

$$
\Omega=\left(\omega^{2}+\omega_{c}^{2} / 4\right)^{1 / 2}
$$

with $\omega_{c}=\mathrm{eB} / \mathrm{m}^{*}$, the cyclotron frecuency. In a magnetic field, the single particle states have the following energies

$$
E_{n m}=\hbar \Omega(n+1)-\frac{1}{2} \hbar \omega_{c} m+s g \mu_{B} B+\alpha B^{2},
$$

where $\mathrm{n}$ is the principal quantum number, $\mathrm{s}$ is the electron spin, $\mathrm{g}$ is the giromagnetic factor, $\mu_{B}$ is the Borh magneton and $\alpha$ is the diamagnetic constant.

The single particle wave functions $\phi$ are parametrized by an effective length, which is denoted as $l_{e}$ for electron and $l_{h}$ for holes. The single particle wave function is given by

$$
\phi_{n m}(r, \theta)=\phi_{m}(\theta) R_{n m}(r),
$$


where

$$
\phi_{m}(\theta)=\frac{1}{\sqrt{2 \pi}} e^{i m \theta}
$$

is the angular part and

$$
R_{n m}(r)=\frac{\sqrt{2}}{l_{e}} \sqrt{\frac{n_{r} !}{\left(n_{r}+|m|\right) !}}\left(\frac{r}{l_{e}}\right)^{|m|} \exp \left[-\frac{r^{2}}{2 l_{e}^{2}}\right] L_{n_{r}}^{|m|}\left(\frac{r^{2}}{l_{e}^{2}}\right)
$$

is the radial part, with $\mathrm{n}_{r}=(\mathrm{n}-|\mathrm{m}|) / 2$.

The matrix element of the Coulomb interaction for a particular state $\phi$ reduces to a sum of integrals involving the basic states. For the interaction between two electrons in basic states $\phi_{i}^{e}$ and $\phi_{j}^{e}$, the integrals are of the form

$$
E_{i j}^{c}=\frac{e^{2}}{4 \pi \varepsilon_{o} \varepsilon_{r}} \iint \frac{\left|\phi_{i}^{e}\left(r_{1}\right)\right|^{2}\left|\phi_{j}^{e}\left(r_{2}\right)\right|^{2}}{\left|r_{1}-r_{2}\right|} d r_{1} d r_{2}
$$

for the direct interactions, and

$$
E_{i j}^{c}=\frac{e^{2}}{4 \pi \varepsilon_{o} \varepsilon_{r}} \iint \frac{\phi_{i}^{e}\left(r_{1}\right)^{*} \phi_{j}^{e}\left(r_{2}\right)^{*} \phi_{i}^{e}\left(r_{2}\right) \phi_{j}^{e}\left(r_{1}\right)}{\left|r_{1}-r_{2}\right|} d r_{1} d r_{2}
$$

for the exchange. These integrals can be evaluated analytically. Similarly, for the interaction between an electron in state $\phi_{i}^{e}$ and a hole in state $\phi_{j}^{h}$, the matrix element of Coulomb interaction is

$$
E_{i j}^{e h}=\frac{e^{2}}{4 \pi \varepsilon_{o} \varepsilon_{r}} \iint \frac{\left|\phi_{i}^{e}\left(r_{e}\right)\right|^{2}\left|\phi_{j}^{h}\left(r_{h}\right)\right|^{2}}{\left|r_{e}-r_{h}\right|} d r_{e} d r_{h} .
$$

This energy can be interpreted as an exciton binding energy: if an electron is in state $\phi_{i}^{e}$ in one dot and a hole in state $\phi_{j}^{h}$ in an identical dot but well separated dot, the total energy is reduced by $\mathrm{E}_{i j}^{e h}$ on confining the carriers in the same dot. The manifestation of tunneling is the modification of the energy spectrum in structures where two or more confined levels are close together in real space. The QDM consists of two QDs separated by a narrow barrier.

\section{RESULTS}

In Fig 1, we show the tunneling of the electron as a function of the separation of the two dots. The inset shows the tunneling of the hole. The tunneling of the hole is shorter than the electron, this in due to the fact that we use heavy hole for our calculations.

Fig. 2 shows the emission energy of the neutral $\mathrm{X}^{0-}$ exciton in a QDM as a function of barrier width. When the dots are not coupled (thick barrier) there are two energy values. These

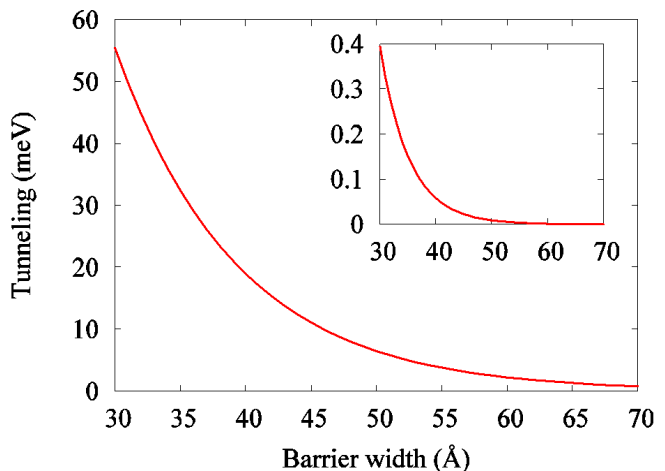

FIG. 1: Electron tunneling as a function of the two dots separation. Inset shows the tunneling of the hole in the same units.

two lines are separated by the $\mathrm{E}_{s s}^{e h}$ according to [6]. For thinner barriers, when the two dots are strongly coupled each line splits and there is an crossing at $\approx 25 \AA$. This splitting is due to the tunneling. The inset shows a QDM with electron and hole in the same dot, as an example of one of the configurations taken into account in the calculation.

We show in Fig. 3 the initial energy of simply charged $\mathrm{X}^{1-}$ exciton as a function of barrier width. For thick barriers we find three eigenenergies, two of them are very closed. They split as the barrier decreases as can be seen in the figure.

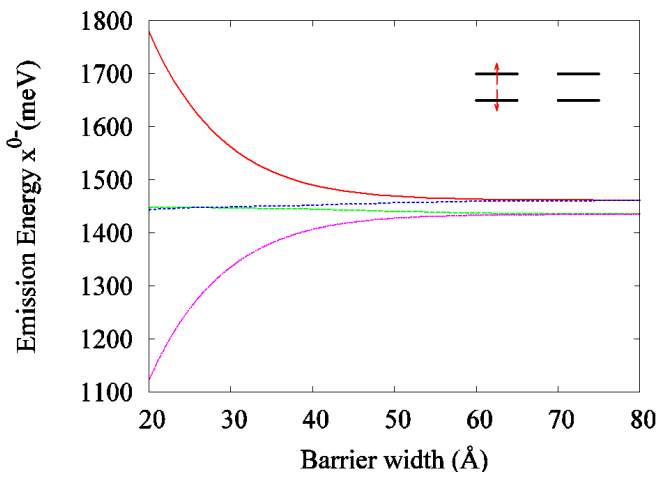

FIG. 2: Emission energy of $\mathrm{X}^{0-}$ exciton in a QDM as a function of barrier with. Inset shows the configuration with electron and hole in the same dot.

In Fig. 4 we show the final energy of $\mathrm{X}^{1-}$ exciton as a function of barrier width. When the barrier is thick, the final energy of the system (one electron) is $\approx 25 \mathrm{meV}$, which corresponds to the chosen confinement. When the barrier decreases, each level splits, as expected.

We show initial energy of $\mathrm{X}^{3-}$ exciton as a function of barrier width in Fig. 5, where we made our calculation with 16 different configurations Here is shown only the four lower energy levels with magnetic field $\mathrm{B}=0$.

Fig. 6 shows the final energy of $\mathrm{X}^{3-}$ exciton as a function of magnetic field in decoupled QDs. The horizontal lines refer to the three particle energy with all electrons into the QDs. 


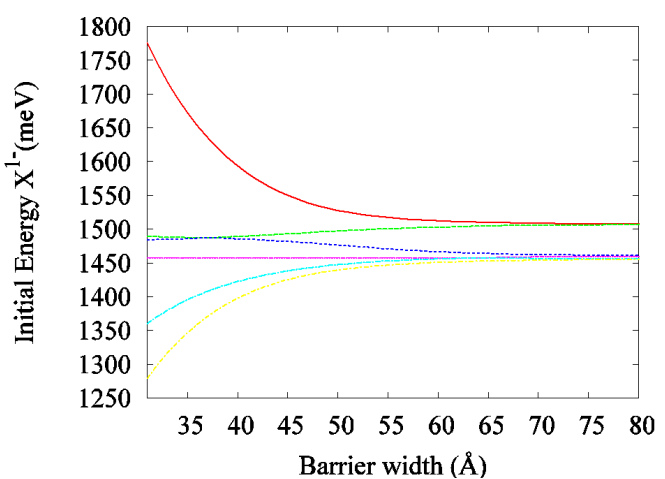

FIG. 3: Initial energy of $\mathrm{X}^{1-}$ exciton in a QDM as a function of barrier with.

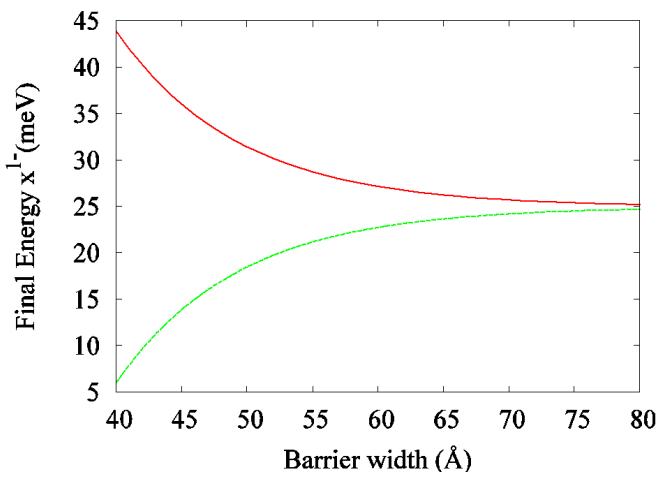

FIG. 4: Final energy of $\mathrm{X}^{1-}$ exciton as a function of barrier width.

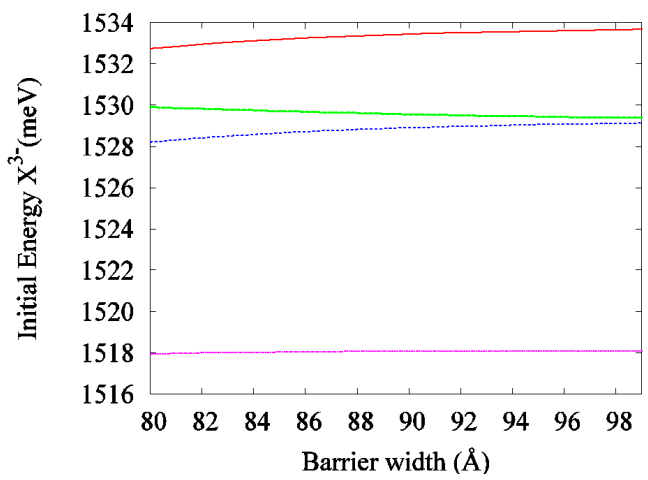

FIG. 5: Initial energy of $\mathrm{X}^{3-}$ exciton as a function of barrier width for the four lowest levels.

Now we analyze the dependence on magnetic field The three lines proportional to magnetic field in Fig. 6 refer to

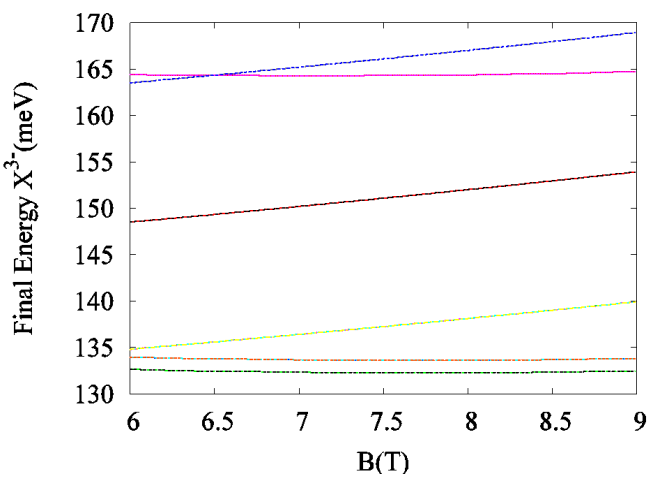

FIG. 6: Final energy of $\mathrm{X}^{3-}$ exciton as a function of magnetic field in decoupled QDs.

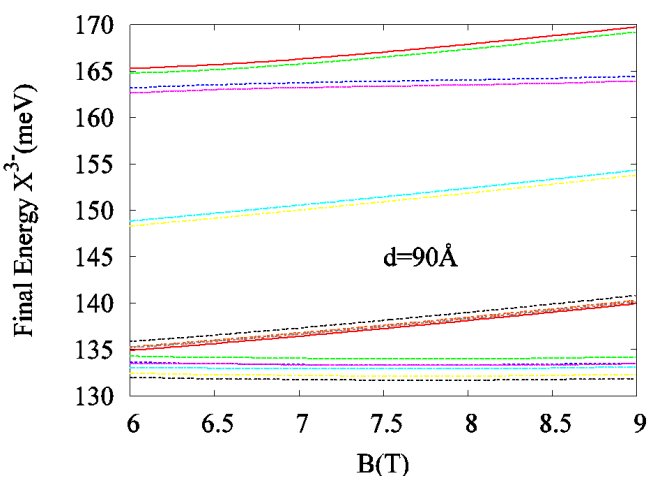

FIG. 7: Final energy of $\mathrm{X}^{3-}$ exciton as a function of magnetic field.

configurations of three particle energy with one of them in the WL. The horizontal lines (independent of field) are the 8 three particle configurations in the dot. Due to the fact that we neglect tunnelling coupling in this figure, we only consider Coulomb interactions between the dots.

We show in Fig. 7. the final energy of $\mathrm{X}^{3-}$ exciton as a function of magnetic field in a QDM taking into account tunneling and also the possible coherent hybridization of localized QD states and extended states. The energies not only split due to tunneling but they also show coupling between the third Landau level and the configuration of two electrons in $\mathrm{p}$ level and one electron in s level through anticrossing at $6,5 \mathrm{~T}$. There are several anticrossing at lower fields that are more difficult to be detected.

For comparison we show in Fig. 8. the final energy of $\mathrm{X}^{3-}$ exciton as a function of magnetic field in a QDM taking into account the coherent hybridization of localized QD states and states of WL at a barrier width of $70 \AA$. We observed a stronger splitting of all levels and also a higher anticrossing energy as should be expected. 


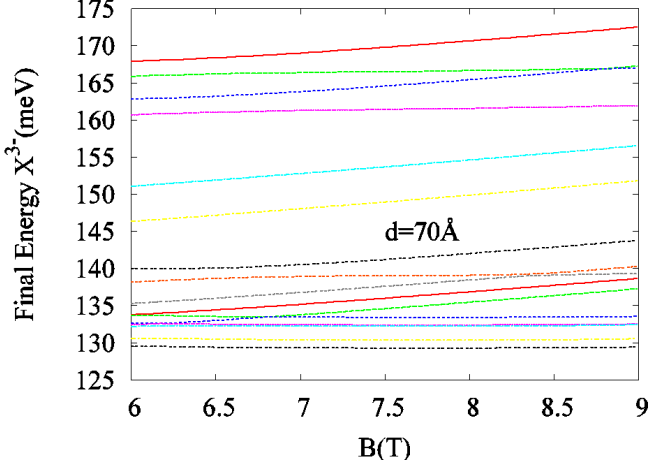

FIG. 8: Final energy of $\mathrm{X}^{3-}$ exciton as a function of magnetic field.

\section{CONCLUSION}

We have presented here, the exciton emission energies in a QDM by using exact diagonalization, for single charge ex- citons as function of barrier width and for triply charged we also calculate its behavior in the presence of an external magnetic field. We find that the Coulomb interaction splits the emission spectra in the charged systems. In the triply charged QDM also the coherent hybridization between localized states with all electrons into the dot and unlocalized states with one electron in the Landau level or WL is taken into account motivated by the results of single QD of reference [2]. We find that the hybridization energy for the molecule is $2,4 \mathrm{meV}$ as in the case of the single QD with the same electronic configuration as the single QD. We also find new anticrossings with the continuum for lower magnetic fields or higher tunneling energies, that are not present in the single dot.

\section{Acknowledgement}

This work was supported by COLCIENCIAS Grant No. 012004.
[1] G. Otner, I. Yugova, G. Baldassarri Höger von Högersthal, A. Larionov, H. Kurtze, D. R. Yakovlev, and M. Bayer, Phys. Rev. B 71, 125335 (2005).

[2] Khaled Karrai, R. J. Warburton, C. Schulhauser, A. Högele, B. Urbaszek, E. J. McGhee, A. O. Govorov, J. M. Garcia, B. D. Gerardot, and P. M. Petroff, Nature 427, 135 (2004).

[3] L. Jacak, P. Hawrylak, and A. Wojs. Quantum Dots (Springer, Berlin, 1998)
[4] R. J. Warburton, B. T. M. Miller, C. S. Dürr, C. Bödefeld, K. Karrai, and J. P. Kotthaus, Phys. Rev. B 58, 16221 (1998).

[5] A. O. Govorov, K. Karrai, and R. J. Warburton, Phys. Rev. B 67, 241307(R) (2003).

[6] M. Bayer, P. Hawrylak, K. Hinzer, S. Fafad, M. Kokusinski, Z. R. Wasilewski, O. Stern, and A. Forchel, Science 291, 451 (2001). 